\title{
Rigidity in Subclasses of Transitive and Mixing Systems
}

\author{
Dawoud Ahmadi Dastjerdi, Maliheh Dabbaghian Amiri \\ The University of Guilan, Rasht, Iran \\ Email: ahmadi@guilan.ac.ir,maliheh@phd.guilan.ac.ir
}

Received July 21, 2012; revised August 26, 2012; accepted September 5, 2012

\begin{abstract}
We will present some restrictions for a rigidity sequence of a nontrivial topological dynamical system. For instance, any finite linear combination of a rigidity sequence by integers has upper Banach density zero. However, there are rigidity sequences for some uniformly rigid systems whose reciprocal sums are infinite. We also show that if $\mathcal{F}$ is a family of subsets of natural numbers whose dual $\mathcal{F}^{*}$ is filter, then a minimal $\mathcal{F}^{*}$-mixing system does not have $F_{+}$-rigid factor for $F \in \mathcal{F}$.
\end{abstract}

Keywords: Rigidity; Filter; Mixing; Upper Banach Density; Density

\section{Introduction}

A topological dynamical system (TDS) is a pair $(X, T)$ such that $X$ is a compact metric space and $T$ is a homeomorphism. Our main concern is rigidity. This notion was first introduced by Furstenberg and Wiess for measure theoretical dynamical system (MDS); then Glasner and Maon defined the topological version of this notion [1].

A comprehensive study for rigidity in MDS has been done in [2]. In MDS, these are interesting; because, it is well-known that a generic transformation is rigid [3]. In this respect and in TDS, Glasner and Maon [1] established examples to show that even in minimal weakly mixing systems, there are plenty of examples with uniformly rigidity.

Let us recall the main definitions. An MDS $(X, \beta, \mu, T)$ is rigid along $\left\{n_{t}\right\}_{t=1}^{\infty}$ if $\left\|f \circ T^{n_{t}}-f\right\| \rightarrow 0$, as $t \rightarrow \infty$ for all $f \in L_{2}(X, \mu)$. A TDS $(X, T)$ is called rigid if there exists a sequence $\left\{n_{t}\right\}_{t=1}^{\infty}$, called the rigidity sequence, such that $T^{n_{t}} X \rightarrow X$ for any $x \in X$; it is called uniformly rigid if $\lim _{t \rightarrow \infty} T^{n_{t}}=\mathrm{id}$ uniformly on $X$.

Let $A \subseteq \mathbb{N}$, then

$$
\bar{d}(A)=\limsup _{n \rightarrow \infty} \frac{|A \cap[1, n]|}{n}
$$

is called the upper density of $A$ and it is called lower density or density if we replace limsup in (1.1) with liminf or lim respectively. We call

$$
d^{*}(A)=\limsup _{(M-N) \rightarrow \infty} \frac{|A \cap[N, M]|}{M-N+1}
$$

the upper Banach density of a set $A \subset \mathbb{N}$.

In a TDS, the return time set is defined to be $N(U, V)=\left\{n \in \mathbb{Z}: T^{n}(U) \cap V \neq \varnothing\right\} \quad$ where $U$ and $V$ are opene (nonempty and open) sets. A TDS $(X, T)$ is transitive if for any two opene sets $U$ and $V$, we have $N(U, V) \neq \varnothing$; and it is weak mixing if the product system $(X \times X, T \times T)$ is transitive. A TDS $(X, T)$ is mild mixing if for any transitive $(Y, S)$, the product system $(X \times Y, T \times S)$ is transitive; and it is strong mixing if $N(U, V)$ is cofinite for opene sets $U, V$.

A collection of subsets of integers $\mathcal{F}$ is called family if it is hereditary upward: if $F_{1} \in \mathcal{F}$ and $F_{1} \subseteq F_{2}$, then $F_{2} \in \mathcal{F}$.

It is well-known that mild mixing systems do not have uniformly rigid factors while minimal equicontinuous systems have comparatively large rigidity sequences. Therefore, one expects to have rigidity along large sequences is system with low complexity. In this note, we define some other classes of mixings. These are defined when $\{N(U, V): U, V$ are opene $\}$ generates a certain family of integers $\mathcal{F}$. In particular, we use this concept and define $\Delta^{*}$-mixings and we show that minimal $\Delta^{*}$ mixings do not have any rigidity factor.

\section{Main Results}

It is well known that in a transitive TDS, any almost equicontinuous is uniformly rigid [1]. In [4] the authors showed that a uniformly rigid mild mixing dynamical system is trivial. Also in [1], Glasner and Maon constructed a generic minimal uniformly rigid weakly mix- 
ing. On the other hand, any system with rigidity sequence has zero entropy [1]. Therefore, a uniformly rigid TDS with zero entropy is generic. However, there are some restrictions for a sequence to be a rigidity sequence. The following shows some of these restrictions which are compatible with the rigidity sequences in MDS [5, Proposition 2.20 (b), 2.24 and 2.26].

Theorem 2.1. Let $A=\left\{n_{t}\right\}$ be an increasing sequence in $\mathbb{N}$ and suppose that for any $k \in \mathbb{N}, T^{k} \neq i d$.

1) If $T$ is rigid along $A$, then $A$ has gaps tending to infinity.

2) Suppose $F\left(x_{1}, \cdots, x_{N}\right)=\sum_{i=1}^{N} c_{i} x_{i}$ where $c_{i} \in \mathbb{Z} \backslash\{0\}$. If for each $M \geq 1$, there is $\left(t_{1}, \cdots, t_{N}\right) \in(\mathbb{N} \backslash\{1, \cdots, M\})^{N}$ such that $F\left(n_{t_{1}}, \cdots, n_{t_{N}}\right)=d$, then $A$ is not a rigidity sequence for any TDS.

3) Suppose $A$ has the property that for some integers $c_{1}, c_{2}, \cdots, c_{L}, d^{*}\left(c_{1} A+\cdots+c_{L} A\right)>0$. Then $A$ cannot be $a$ rigidity sequence for a TDS. In particular, $d^{*}(A)=0$.

Proof. We prove only (1) and the two others follow similarly. Let $(X, T)$ be rigid along $\left\{n_{t}\right\}$. Then $T^{n_{t}} X \rightarrow x$ for every $x \in X$. By the dominated convergence theorem for every invariant measure and in particular for ergodic measure $\mu$ and any $f \in L_{2}(\mu)$ we have $\lim T^{n_{t}} f=f$. This shows that $(X, T, \mu)$ is rigid along $\left\{n_{t}\right\}$ in measure theoretical sense. By [5, Proposition $2.20(\mathrm{~b})]$, if $\left\{n_{t}\right\}$ is a rigidity sequence for ergodic $(X, T)$, then $\left\{n_{t}\right\}$ has gaps tending to infinity.

Note that the second part of the conclusion in (2) follows from the fact that sets having positive upper Banach density have a certain distance appearing infinitely many times.

If $A=\left\{n^{2}: n \geq 0\right\}$, then $\{2 n+1: n \in \mathbb{N}\} \subseteq A-A$ and so it has positive density. Therefore, $A$ cannot be a uniformly rigidity sequence for any $(X, T)$. This is also true for sequence of prime numbers and polynomial sequence with integer coefficients.

Let

$$
\Sigma=\left\{A \subset \mathbb{N}: \sum_{a \in A} \frac{1}{a}=\infty\right\} .
$$

From largeness point of view, $\Sigma$ is next to the family of positive upper density, that is, if $\bar{d}(B)>0$ then $B \in \Sigma$ [6]. This family has many interesting properties and it is a long standing conjecture by Erdös that any member of this family has arbitrary long arithmetic progression. In the following example we show that there are some uniformly rigid TDS whose rigidity sequence is in $\Sigma$.

Example 2.2. Let $X=S^{1}$ and $T$ the irrational rotation on $S^{1}$ (or consider any equicontinuous minimal system). Note that for any $X=x \in X$ and any opene set $U$, the return time set $N(x, U)$ is syndetic and if rigidity is established for a point $x_{0}$, that is if there exists $\left\{n_{t}\right\}$ such that $T^{n_{t}}\left(x_{0}\right) \rightarrow x_{0}$, then rigidity is established for all points. Also rigidity and uniform rigidity are equivalent for our system.

First we construct a rigidity sequence $\left\{n_{t}\right\}$ and then we will show that $A=\left\{n_{t+1}-n_{t}\right\}$ which is trivially a rigidity sequence is in $\Sigma$. So let $x_{0}=0$ and let $\left\{\epsilon_{i}\right\}$ be a decreasing sequence to zero and set $\left\{b_{i}\right\}$ to be the sequence with $b_{i}$ the maximum gap for $N\left(0, B_{\epsilon_{i}}(0)\right)$.

Set $l_{0}:=0, k_{1}:=l_{1}-l_{0}>b_{1}$ and pick consecutive $n_{1}, n_{2}, \cdots, n_{l_{1}} \in N\left(0, B_{\epsilon_{i}}(0)\right)$. For any $1 \leq i \leq l_{1-1}$ we have $n_{i+1}-n_{i} \leq b_{1}$ and $\frac{k_{1}}{b_{1}}<\sum_{i=1}^{l_{1}-1} \frac{1}{n_{i+1}-n_{i}}$.

Use induction argument and let $n_{l_{i}+1}, \ldots, n_{l_{i+1}} \in N\left(0, B_{\epsilon_{i}}(0)\right)$ such that $k_{i+1}:=l_{i+1}-l_{i}>b_{i+1}$. So $\frac{k_{i+1}}{b_{i+1}}<\sum_{t=l_{i}+1}^{l_{i+1-1}} \frac{1}{n_{t+1}-n_{t}} \quad$ and thus $\sum_{i \geq 1} \frac{k_{i}}{b_{i}}<\sum_{t \geq 1} \frac{1}{n_{t+1}-n_{t}}$. But for any $i, \frac{k_{i}}{b_{i}}>1$ which implies $\sum_{i \geq 1} \frac{1}{n_{i+1}-n_{i}}=\infty$.

Remark 2.3.1) Let $D(A)=A-A$. Then $D^{2}(A)=D(D(A))$ and in general $D^{n}(A)$ can be defined for any $n \in \mathbb{N}$. In [7], for any $n \in \mathbb{N}$, an explicit subset of $\mathbb{N}$ such as $A \in \Sigma$ depending on $n$ is given such that $d^{*}\left(D^{n}(A)\right)=0$. Now the existence of such sets is established by the above example and Theorem 2.1(3). In fact, we have more: there is $B \in \Sigma$ such that for any $n \in \mathbb{N}, d^{*}\left(D^{n}(B)\right)=0$.

2) If $T^{n_{t}}(x) \rightarrow \tau(x)$ along a subsequence $\left\{n_{t}\right\}$, then $T$ is rigid along $A=\left\{n_{t+1}-n_{t}\right\}$ and it is uniform rigid if $T^{n_{t}}(x) \rightarrow \tau(x)$ is uniform. If $S$ is also rigid along $A$, then $T \times S$ is rigid and it is uniform if both $T$ and $S$ are uniformly rigid along $A$.

Theorem 2.4. Suppose $(X, T)$ is rigid along $\left\{n_{t}\right\}$ and $(Y, S)$ a TDS. Then $(X \times Y, T \times S)$ is rigid if there exists $c_{1}, \cdots, c_{k} \in \mathbb{Z}$ and $N_{1}, N_{2}, \cdots, N_{k} \in \mathbb{N} \bigcup\{0\}$ such that $(Y, S)$ is rigid along $\left\{\sum_{i=1}^{k} c_{i} n_{t}+N_{i}\right\}_{t \geq 1}$.

Proof. If $(X, T)$ is rigid along $\left\{n_{t}\right\}$, then it is also rigid along $\left\{\sum_{i=1}^{k} c_{i} n_{t}+N_{i}\right\}_{t \geq 1}$ for any $c_{1}, \cdots, c_{k} \in \mathbb{Z}$ and $N_{1}, N_{2}, \cdots, N_{k} \in \mathbb{N} \bigcup\{0\}$.

Corollary 2.5. Let $(G, \rho)$ be a rotation and $(X, T)$ a rigid (resp. uniformly rigid) system. Then

$(G \times X, \rho \times T)$ is rigid (resp. uniformly rigid).

Proof. Suppose $(X, T)$ is rigid along some sequence $\left\{n_{t}\right\}$. Let $\rho(x)=x+\alpha$ be the rotation map. For any $\left\{n_{t}\right\}$, by passing to a subsequence if necessary, we have 
$n_{t} \alpha \rightarrow \beta$ for some $\beta \in G$. This means that $\rho^{n_{t}} \rightarrow S$ where $S(x)=x+\beta$. Hence $\rho^{n_{t+1}-n_{t}} \rightarrow S \circ S^{-1}=$ id uniformly and $\left(\rho^{n_{t+1}-n_{t}}, T\right) \rightarrow\left(\mathrm{id}_{\rho}, \mathrm{id}_{T}\right)$.

Theorem 2.6. Suppose $(X, T)$ is rigid. Then any factor is rigid.

Proof. This is clearly true for the trivial factor. So let $(X, T)$ be rigid along $\left\{n_{t}\right\}_{t \in \mathbb{Z}}$ and $(Y, S)$ a nontrivial factor with factor map $\pi: X \rightarrow Y$. We show that $(Y, S)$ is rigid along a subsequence of $\left\{n_{t}\right\}$. To this end, let $y$ be an arbitrary point in $Y, x=\pi^{-1} y$ and $V$ an opene set containing $y$. Since $(X, T)$ is rigid there exists $N \in \mathbb{N}$ such that for any $t>N, T^{n_{t}}(x) \in \pi^{-1} V$. Thus $\pi\left(T^{n_{t}} x\right) \in V$ and so $S^{n_{t}} y \in V$.

Let $\mathcal{F}$ be a family of nonempty subsets of $\mathbb{Z}$. The dual of $\mathcal{F}$, denoted by $\mathcal{F}^{*}$, is defined to be all subsets of $\mathbb{Z}$ meeting all sets in $\mathcal{F}$ :

$$
\mathcal{F}^{*}=\{G \subset \mathbb{Z}: G \bigcap F \neq \varnothing, \forall F \in \mathcal{F}\} .
$$

A family $\mathcal{F}$ is called partition regular if $F \in \mathcal{F}$ is partitioned to finite sets $F=F_{1} \cup \cdots \cup F_{k}$, then there is $1 \leq i \leq k$ such that $F_{i} \in \mathcal{F}$. An example of a family with partition regularity is the family $\Sigma$ defined as (2.1). A nonempty family closed under finite intersections is called a filter. It is known that if $\mathcal{F}$ is partition regular, then $\mathcal{F}^{*}$ is a filter. A filter which is partition regular is called an ultrafilter.

Now let $A=\left\{a_{n}\right\}_{n \in \mathbb{N}}$ be an increasing sequence of integers. Then $F S(A)=\left\{a_{i_{1}}+a_{i_{2}}+\cdots+a_{i_{n}}: i_{j}<i_{j+1}\right\}$ is the finite sums of $A$. A set $F \subseteq \mathbb{Z}$ is called an IP-set if it contains the finite sums of some sequence of integers. A set $F \subset \mathbb{Z}$ is called a $\Delta$-set if a sequence of integers $S=\left(s_{n}\right)_{n \in \mathbb{N}}$ exists such that the difference set $\Delta(S)=\left\{s_{i}-s_{j}: i>j\right\} \subset F$. Let $\Delta$ be the family of all $\Delta$-sets. Any IP-set is a $\Delta$-set for let

$S=\left\{a_{1}, a_{1}+a_{2}, a_{1}+a_{2}+a_{3}, \cdots\right\}$. Let $\mathcal{I P}$ (resp. $\Delta$ ) be the family of all IP-sets (resp. $\Delta$-sets). It is known that the families $\mathcal{I P}^{*}$ and $\Delta^{*}$ are filters [8].

Definition 2.7. A TDS $(X, T)$ is called $\mathcal{F}$-transitive if for any two opene sets $U, V \subset X$ we have $N(U, V) \in \mathcal{F}$, and it is called $\mathcal{F}$-mixing if the product system $(X \times X, T \times T)$ is $\mathcal{F}$-transitive.

Theorem 2.8. [9] Let $(X, T)$ be a TDS. The following conditions are equivalent:

1) $(X, T)$ is $\mathcal{F}$-mixing;

2) $(X, T)$ is weak mixing and $\mathcal{F}$-transitive;

3) $N(U, V) \cap N(U, U) \in \mathcal{F}$ for any opene sets $U, V$.

For a family $\mathcal{F}$ and $k \in \mathbb{Z}$, the shifted family is defined as $\mathcal{F}+k=\{F+k: F \in \mathcal{F}\}$ where

$F+k=\{n+k: n \in F\}$. If $\mathcal{F}+k=\mathcal{F}$ for any $k \in \mathbb{Z}$, then $\mathcal{F}$ is called a shift invariant family. For instance, if $\mathcal{I}=\{F \subseteq \mathbb{Z}:|F|=\infty\}$, then both $\mathcal{I}$ and $\mathcal{I}^{*}$ are shift invariant families. But not all families are shift in- variant. There are two ways to build a shift invariant family from a given $\mathcal{F}$ [8]. These are $\mathcal{F}_{+}$and $\mathcal{F}$. where

$$
\mathcal{F}_{+}:=\bigcup_{k \in \mathbb{Z}}(\mathcal{F}+k), \mathcal{F}_{.}:=\bigcap_{k \in \mathbb{Z}}(\mathcal{F}+k) .
$$

We have $\mathcal{F}_{\text {. }} \subset \mathcal{F} \subset \mathcal{F}_{+}$and both $\mathcal{F}_{\text {. }}$ and $\mathcal{F}_{+}$are shift invariant families with $\mathcal{F}^{*}=\left(\mathcal{F}_{+}\right)^{*}$ [8]. Also, if $\mathcal{F} \subset \mathcal{F}^{\prime}$ then $\mathcal{F}_{+} \subset \mathcal{F}_{+}^{\prime}$ and $\mathcal{F}^{*} \subset \mathcal{F}^{*}$ which implies that $\mathcal{F}^{\prime *} \subset \mathcal{F}^{*}$. If $\mathcal{F}$ is a filter so is any shift of $\mathcal{F}$ and since the finite intersections of filters are again filters $\mathcal{F}$. is a filter.

Theorem 2.9. 1) $(X, T)$ is $\mathcal{F}$.-transitive if and only if it is $\mathcal{F}$-transitive.

2) Suppose $\mathcal{F}$ is a filter. Then $(X, T)$ is $\mathcal{F}$. - mix ing if and only if it is $\mathcal{F}$-mixing.

Proof. 1) We have $\mathcal{F} . \subseteq \mathcal{F}$, so $\mathcal{F}$. -transitive is $\mathcal{F}$ transitive. Conversely, suppose $(X, T)$ is $\mathcal{F}$-transitive. Then for any opene $U$ and $V$, we have $N(U, V) \in \mathcal{F}$. Since $T^{-k} U$ is opene for $k \in \mathbb{Z}$, $N\left(T^{-k} U, V\right)=N(U, V)+k \in \mathcal{F}$. This means that for $k \in \mathbb{Z}, \quad N(U, V)+k \in \mathcal{F}$ which in turn implies $N(U, V) \in \mathcal{F}_{\text {. }}$

2) This is a direct consequence of the first part and Theorem 2.8 .

Theorem 2.10. Let $\mathcal{F}$ be a filter and $(X, T)$ an $\mathcal{F}$ mixing system. Then any non-trivial factor of $(X, T)$ is also $\mathcal{F}$-mixing.

Proof. Suppose $(X, T)$ is $\mathcal{F}$-mixing and $(Y, S)$ a non-trivial factor and $\phi$ the factor map: $\phi \circ T=S \circ \phi$. For any two opene sets $U, V \subset X$,

$N(U, V) \cap N(U, U) \in \mathcal{F}$. We will show that this will hold for $(Y, S)$ as well.

Let $U^{\prime}, V^{\prime}$ be two opene sets in $Y$ and let $U, V \subset X$ such that $U^{\prime}=\phi(U), V^{\prime}=\phi(V)$. Then

$$
\begin{aligned}
N\left(U^{\prime}, V^{\prime}\right) & =\left\{n: S^{n}(\phi U) \cap \phi V \neq \varnothing\right\} \\
& =\left\{n: \phi\left(T^{n} U\right) \cap \phi V \neq \varnothing\right\} \\
& \supseteq\left\{n: \phi\left(T^{n} U \cap V\right) \neq \varnothing\right\} \\
& =\left\{n: T^{n} U \cap V \neq \varnothing\right\}=N(U, V) \in \mathcal{F} .
\end{aligned}
$$

Since $\mathcal{F}$ is a family, so $N\left(U^{\prime}, V^{\prime}\right) \in \mathcal{F}$. Also, since $\mathcal{F}$ is a filter $N\left(U^{\prime}, V^{\prime}\right) \cap N\left(U^{\prime}, U^{\prime}\right) \in \mathcal{F}$ which implies $(Y, S)$ is $\mathcal{F}$-mixing.

Let $\mathcal{F}$ be a family of subsets of integers closed under finite intersections (in general like a filter). Then we say that a sequence $\left\{x_{n}\right\}$ is $\mathcal{F}$-convergent to $x$ if for any neighborhood $U$ of $x$ we have $\left\{n \in \mathbb{Z}: T^{n}(x) \in U\right\} \in \mathcal{F}$ and we write $\mathcal{F}-\lim _{n \rightarrow \infty} x_{n}=x$. A family is called an $\mathcal{I P}-\mathcal{I P}$ family if any member contains the difference set of an IP-set.

Theorem 2.11. Let $(X, T)$ be uniformly rigid along 
$A=\left\{a_{n}\right\}_{n \geq 1}$. Then $(\mathcal{I P}-\mathcal{I P})-\lim _{n \rightarrow \infty} T^{n}=i d$.

Proof. First we prove that $\mathcal{I P}-\lim T^{n}=$ id. Let $(X, T)$ be uniformly rigid along $\stackrel{n \rightarrow \infty}{=}\left\{a_{n}\right\}_{n \geq 1}$. Fix $\epsilon>0$ and let $N_{k} \in \mathbb{N}$ be an increasing sequence and each $N_{k}$ sufficiently large so that

$$
d\left(T^{a_{N_{k}}}, \mathrm{id}\right)<\frac{\epsilon}{2^{k}}
$$

Hence for any $i_{1}, \cdots, i_{k}$. we have

$$
\begin{aligned}
& d\left(T^{a_{N_{i 1}}+\cdots+a_{N_{i k}}}, \mathrm{id}\right) \\
& \leq d\left(T^{a_{N_{i_{1}}}}\left(T^{a_{N_{i_{2}}}+\cdots+a_{N_{i_{k}}}}\right),\left(T^{a_{N_{i_{2}}}+\cdots+a_{N_{i_{k}}}}\right)\right) \\
& +d\left(T^{a_{N_{i_{2}}}}\left(T^{a_{N_{i_{3}}+\cdots+a_{N_{i_{k}}}}}\right),\left(T^{a_{N_{i_{3}}}+\cdots+a_{N_{i_{k}}}}\right)\right) \\
& +\cdots+d\left(T^{a_{N_{i_{k}}}}, \mathrm{id}\right) \\
& <\sum_{n=1}^{k} \frac{\epsilon}{2^{i_{n}}}<\epsilon \text {. }
\end{aligned}
$$

Now set $B:=F S\left(a_{N_{i}}\right)$. Then $\left\{n: d\left(T^{n}\right.\right.$, id $\left.)<\epsilon\right\}$ contains $B$ and so is an IP-set.

To see that $(\mathcal{I P}-\mathcal{I P})-\lim _{n \rightarrow \infty} T^{n}=\mathrm{id}$, note that if $\left\{n_{t}\right\}$ is a rigidity sequence so is $\left\{-n_{t}\right\}$. Then an inequality such as (2.2) implies that $d\left(T^{\alpha-\beta}\right.$, id $)<\epsilon$ where $\alpha, \beta \in B$.

Now we investigate the existence of rigidity (not necessarily uniform) in minimal systems with some sort of mixings. Recall that a minimal system is mild mixing if and only if it is $\mathcal{I P}{ }^{*}$-mixing if and only if it is $(\mathcal{I P}-\mathcal{I P})^{*}$-transitive [10].

A pair $(x, y) \in X \times X$ is said to be a proximal pair if

$$
\liminf _{n \rightarrow \infty} d\left(T^{n}(x), T^{n}(y)\right)=0
$$

and $(X, T)$ is proximal system if any pair of $X \times X$ is a proximal pair. A TDS $(X, T)$ is called distal if $\liminf _{n \rightarrow \infty} d\left(T^{n}(x), T^{n}(y)\right)>0$ for every $x \neq y$ and $(x, y) \in X \times X$. In [1], the authors showed that any minimal strong mixing system admits only trivial rigid factors. An extension of that result is the following.

Theorem 2.12. Suppose $\mathcal{F}^{*}$ is a filter. Then a minimal $\mathcal{F}^{*}$-mixing system does not have $F_{+}$-rigid factor where $F \in \mathcal{F}$.

Proof. Let $(X, T)$ be a minimal $\mathcal{F}^{*}$-mixing. Then by Theorem 2.9 and Theorem 2.10, every factor of $(X, T)$ is $\mathcal{F}_{\text {. }}^{*}$-mixing. Thus it is sufficient to show that if $(X, T)$ is $F_{+}$-rigid, then it must be trivial. Assume that $(X, T)$ is rigid with respect to an $F_{+}$sequence $\left\{n_{k}\right\}$. Let $\left(x, x^{\prime}\right) \in\left(U_{k}, V_{k}\right) \subset X \times X$, where $U_{k}=B\left(x, \frac{1}{k}\right)$ and $V_{k}=B\left(x^{\prime}, \frac{1}{k}\right)$. Note that $N\left(U_{k}, V_{k}\right)$ is $\mathcal{F}_{\text {. }}^{*}$ and so $\left|N\left(U_{k}, V_{k}\right) \cap F_{+}\right|=\infty$. Therefore, there exists a subsequence $\left\{n_{k}^{\prime}\right\}$ of $\left\{n_{k}\right\}$ such that $x_{k} \in T^{-n_{k}^{\prime}}\left(T^{n_{k}^{\prime}} U_{k} \cap V_{k}\right), x_{k} \rightarrow x$ and $T^{n_{k}^{\prime}} x_{k} \rightarrow x^{\prime}$. Which implies that $\left(x, x^{\prime}\right)$ is a proximal pair. Since $\left(x, x^{\prime}\right)$ was arbitrary the system is proximal. But in a minimal system, $(x, T x)$ is distal for any $x$ and this in turn implies that $(X, T)$ must be trivial.

Corollary 2.13. 1) A minimal $\Delta^{*}$-mixing system does not have any rigid factor.

2) A minimal $(\Delta-\Delta)^{*}$-transitive system is not rigid.

3) Any $\Delta^{*}$-mixing, $I P^{*}$-mixing or $(\Delta-\Delta)^{*}$-transitive system does not have a non-trivial uniformly rigid factor.

Proof. 1) By Theorem 2.10, it suffices to show that a minimal $\Delta^{*}$-mixing $(X, T)$ is not rigid along any $\left\{n_{t}\right\}$. Assume the contrary and let $\left\{\epsilon_{k}\right\}$ be a sequence decreasing to zero. Let $\left(x, x^{\prime}\right) \in\left(U_{k}, V_{k}\right) \subset X \times X$, where $U_{k}, V_{k}$ are $\epsilon_{k}$-balls containing $x$ and $x^{\prime}$ respectively. Then there exists $M_{k}>0$ such that for any $a \in A_{k}:=\Delta\left(\left\{n_{t}\right\}_{t>M_{k}}\right), d\left(T^{a} x, x\right)<\epsilon_{k}$. Since $(X, T)$ is $\Delta^{*}, N\left(U_{k}, V_{k}\right) \cap A_{k} \neq \varnothing$. Now if $\epsilon_{k} \rightarrow 0$, then there exists a subsequence $\left\{a_{k}\right\}, a_{k} \in A_{k}$ and a sequence $x_{k} \rightarrow x$ such that $T^{a_{k}} x_{k} \rightarrow x^{\prime}$ and $T^{a_{k}} x \rightarrow x$. Now an argument as in the proof of Theorem 2.12 gives the proof.

2) The proof is similar to (1).

3) Recall that any $\Delta^{*}$-mixing, $I P^{*}$-mixing and $(\Delta-\Delta)^{*}$-transitive is trivially an $(\mathcal{I P}-\mathcal{I P})^{*}$-transitive. Now the conclusion follows from the fact that mild mixing systems do not have non-trivial uniform rigid factors.

\section{REFERENCES}

[1] S. Glasner and D. Maon, "Rigidity in Topological Dynamics," Ergodic Theory and Dynamical Systems, Vol. 9, No. 2, 1989, pp. 309-320.

[2] H. Furstenberg and B. Wiess, "The Finite Multipliers of Finite Transformation," Lecture Note in Math, Vol. 688 1978, pp. 127-132.

[3] A. Katok and A. Stepin, "Approximation in Ergodic Theory," Russian Mathematical Surveys, Vol. 22, No. 5, 1967, pp. 77-102. doi:10.1070/RM1967v022n05ABEH001227

[4] E. Glasner and D. Maon, "On the Interplay between Measure and Topolgical Dynamics," In: Hasselblatt and Katok, Eds., Hand Book of Dynamical System, Elsevier, Amsterdam, 2006, pp. 597-648.

[5] V. Bergelson, A. Del Junco, M. Lemanczyk and J. Rosenblatt, "Rigidity and Non-Recurrence along Sequences," arXive: $1103.0905 \mathrm{v} 1$ [math. DS].

[6] T. C. Brown and A. R. Freedman, "Arithmetic Progression in Lacunary Sets," Rocky Mountain, Journal of 
Mathematics, Vol. 17, No. 17, 1987, pp. 578-596.

[7] D. A. Dastjerdi and M. Hosseini, "Difference Sets of Null Density Subsets of $\mathbb{N}$," Advances in Pure Mathematics, Vol. 2, No. 3, 2012, pp. 195-199. doi:10.4236/apm.2012.23027

[8] V. Bergelson and T. Downarowicz, "Large Sets of Integers and Hierarchy of Mixing Properties of Measure-Preserving Systems," Colloquium Mathematicum, Vol. 110,
No. 1, 2008, pp. 117-150. doi:10.4064/cm110-1-4

[9] W. Huang, S. Shao and X. Ye, "Mixing via Sequence Entropy," Contemporary Mathematics, Vol. 385, 2005, pp. 101-122. doi: $10.1090 / \mathrm{conm} / 385 / 07193$

[10] W. Huang and X. Ye, "Topological Complexity, Return Times and Weak Disjointness," Ergodic Theory and Dynamical Systems, Vol. 24, No. 3, 2004, pp. 825-846. doi:10.1017/S0143385703000543 